\title{
Lack of association between the vitamin $D$ receptor polymorphism rs2228570 and chronic periodontitis in a Han Chinese population
}

\author{
X. Wang ${ }^{1}$, T.L. Zhang ${ }^{2}$ and D. Chen ${ }^{3}$ \\ ${ }^{1}$ School of Public Health, Weifang Medical University, Weifang, China \\ ${ }^{2}$ Medicine Research Center, Weifang Medical University, Weifang, China \\ ${ }^{3}$ Department of Periodontology, Shanghai Stomatological Disease Center, \\ Shanghai, China \\ Corresponding author: D. Chen \\ E-mail: chendong19690124@126.com
}

Genet. Mol. Res. 14 (4): 12299-12305 (2015)

Received March 10, 2015

Accepted July 13, 2015

Published October 9, 2015

DOI http://dx.doi.org/10.4238/2015.October.9.18

\begin{abstract}
The vitamin D receptor (VDR) is involved in the immune response and bone metabolism, both of which are implicated in the pathogenesis of chronic periodontitis (CP). In this study, we investigated the association between the VDR single nucleotide polymorphism rs2228570 and $\mathrm{CP}$ susceptibility in a Han Chinese population consisting of 440 moderate or severe CP patients and 324 controls. Genomic DNA was extracted from buccal epithelial cells and genotyped using matrix-assisted laser desorption/ ionization time-of-flight-mass spectrometry. No significant difference in allelic or genotypic frequencies distributions for rs2228570 was found between $\mathrm{CP}$ patients and controls. In conclusion, we found no statistically significant association between rs2228570 and CP in our population.
\end{abstract}

Key words: Chronic periodontitis; Single nucleotide polymorphism; Vitamin $D$ receptor 


\section{INTRODUCTION}

Periodontitis, which is initiated by microbial plaque accumulation at a gingival crevice, is a type of chronic inflammatory disease affected by both environmental and genetic factors (Stabholz et al., 2010). Chronic periodontitis (CP), the most common form of periodontitis affecting up to $30 \%$ of adults (Nares, 2003), is characterized by slow progressing alveolar bone resorption and attachment loss (Armitage, 1999). It has been reported that nearly $50 \%$ of periodontal conditions [attachment loss, pocket depth (PD), gingival index, and plaque index] measures varied in 110 adult twins with a mean age of 40 years ranging from 16 to 70 years; thus, genetic factors contribute to differences in CP (Michalowicz et al., 2000). Most of the investigated candidate genes related to the periodontitis susceptibility mainly included those implicated in the host immune response and in the vitamin D receptor (VDR) gene (Kinane et al., 2005).

VDR, also known as 1,25-dihydroxyvitamin D3 receptor, is located on chromosome $12 q 13.11$ and belongs to a superfamily of nuclear receptors that function as transcription factors and play a vital role in the vitamin D pathway (OMIM, 2014). VDR is a transcriptional regulator of vitamin D-responsive genes. The vitamin $D$ endocrine system is important in numerous biological processes, such as bone metabolism and modulation of immune response (Uitterlinden et al., 2004). Bone is constantly remodeling through the cross-regulation of osteoclasts and osteoblasts, both of which are responsible for bone resorption and formation, respectively. Vitamin $D$ induces osteoclast differentiation and stimulates bone resorption, resulting in calcium release from the bone in case of need, which is known to be mediated by VDR through a signaling pathway coupling osteoblasts and osteoclasts. The functions of vitamin $D$ in both infection prevention and calcium metabolism disorders have been demonstrated in VDR knockout mice (Nagpal et al., 2005). Moreover, enamel and dentin abnormalities, which are related to dental resorption, were observed in VDR null mice (Descroix et al., 2010).

The VDR single nucleotide polymorphism (SNP) rs2228570 was reported to be associated with severe CP in a Japanese population (Naito et al., 2007). A previous linkage disequilibrium (LD) analysis of SNPs located in the VDR gene demonstrated that rs2228570 was not in LD with any other SNP (Nejentsev et al., 2004). Because of the vital dual roles of VDR in the immune response and in bone metabolism, both are implicated in the pathogenesis of CP. We hypothesized that rs2228570 may be a candidate gene locus for CP susceptibility in Chinese subjects. In this study, we investigated whether the SNP rs2228570 is associated with CP susceptibility in a Han Chinese population.

\section{MATERIAL AND METHODS}

\section{Study population and clinical assessments}

Subjects from Shanghai area of China were recruited from the patients' database of Department of Periodontology, Shanghai Stomatological Disease Center between December 2009 and December 2011. The study was reviewed and approved by the Shanghai Stomatological Disease Center ethics committee (date of approval: September 14, 2009; approval number: 2009005). A total of 440 unrelated Han Chinese patients diagnosed with moderate CP (mean age, 49.9 years) or severe CP (mean age, 46.6 years) and 324 ethnically matched with control participants (mean age 50.4 years) in the present study by random selection. All participants signed written 
informed consent after learning the relevant information about the study. No subjects displayed significant differences in the ratio between men and women or ages among the groups. The study was conducted in full accordance with ethical principles, including the World Medical Association Declaration of Helsinki (version 2002) and additional requirements in China.

Included CP patients met the following criteria: 1) at least 10 teeth lost, i.e., no more than 18 existing teeth; or 2) at least 2 interproximal sites (not on the same tooth) with clinical attachment loss (CAL) $\geq 6 \mathrm{~mm}$ and at least 1 interproximal site with pocket depth $\geq 5 \mathrm{~mm}$; or 3 ) at least 2 interproximal sites with $C A L \geq 4 \mathrm{~mm}$ (not on the same tooth) or at least 2 interproximal sites with PD (not on the same tooth) $\geq 5 \mathrm{~mm}$. In addition, the percentage of PD $\geq 4 \mathrm{~mm}$ should be in the upper tertile of the participants, meaning that the percentage of the sites of PD $\geq 4 \mathrm{~mm}$ should be more than $33 \%$ in all PD sites ( 6 sites altogether examined on each tooth) in one patient's mouth. These criteria were modified from the definitions proposed by the CDC/AAP working group (Eke et al., 2012). The CP patients included also met the criteria proposed by the Community Periodontal Index of Treatment Needs in addition to those of the CDC/AAP working group. According to these proposed procedures, 10 index teeth $(11,16,17,26,27,31,36,37,46,47)$ for each subject were examined and periodontal PD and/or Community Periodontal Index of Treatment Needs index scores were recorded. The measurements used in the severity classification of periodontal disease were based on the worst affected index tooth for each parameter such as CAL. Patients with $C A L \geq 3$ to $<5 \mathrm{~mm}$ were considered as moderate, while those with $C A L \geq 5 \mathrm{~mm}$ were considered as severe CP. None of the included healthy control participants has periodontal disease as determined by CAL, PD $<3 \mathrm{~mm}$, or attachment loss or no alveolar bone loss by X-ray.

Participants with a history or current signs of any systemic or oral disease other than periodontitis, those who were pregnant or lactating, and those receiving periodontal or antibiotic treatment within 6 months prior to the study were excluded from the present study. Subjects who were tobacco users, whether in a smoking or smokeless form, were also excluded. The participants' buccal epithelial samples were collected for subsequent laboratory analysis.

\section{DNA extraction}

Buccal epithelial cells were collected from the participants and genomic DNA extraction was conducted using the phenol/chloroform and salt/ethanol precipitation. The genomic DNA was quantified by absorbance at $260 \mathrm{~nm}$ using a spectrophotometer (GE Healthcare, Little Chalfont, UK), dissolved in buffer containing $10 \mathrm{mM}$ Tris, $\mathrm{pH} 7.8$ and $1 \mathrm{mM}$ EDTA, and then stored at $-70^{\circ} \mathrm{C}$ until use.

\section{SNP genotyping}

The primers used for the rs2228570 polymerase chain reaction and genotyping were as follows: ACGTTGGATGTGGCCTGCTTGCTGTTCTTA (forward), ACGTTGGATGAAGTCTCCAGGGTCAGGCA (reverse), and CCCTTCTTGCTGTTCTTACAGGGA (single-base extension primer). The genotyping of rs2228570 was performed using a matrixassisted laser desorption/ionization time-of-flight-mass spectrometry method as described previously (Schaeffeler et al., 2008). SNP genotyping was performed using the MassARRAY system (Sequenom, San Diego, CA, USA). The genotyping reactions were performed using a 384-well spectroCHIP (Sequenom) equipped with a MassARRAY Nanodispenser. Genotype calling was carried out using the MassARRAY RT software (version 3.1, Sequenom) in a real-time 
monitoring manner. The data were analyzed using the MassARRAY Typer software (version 4.0, Sequenom). For genotyping quality control, $10 \%$ of genomic DNA samples from the participants were randomly selected and genotyped twice; the repeated genotyping showed no discrepancies. Furthermore, in case of bias, all genomic DNA samples used for genotyping were coded randomly without revealing any relevant information of the donors.

\section{Statistical analysis}

Quantitative parameters are reported as means \pm SD unless indicated otherwise. Whether genotypes of rs2228570 were in accordance with Hardy-Weinberg equilibrium was determined using the chi-square test by comparing the observed allele frequencies with expected allele frequencies. To measure the association between CP susceptibility and rs2228570 alleles or genotypes, contingency table analysis and chi-square test were used for odds ratio and $95 \%$ confidence intervals calculation, followed by logistic regression to adjust for the effects of age and gender. All $P$ values were 2-sided and values were considered statistically significant when the $P$ value was $<0.05$. All statistical analyses were performed using the SPSS 19.0 software (SPSS, Inc., Chicago, IL, USA).

\section{RESULTS}

The demographic features of patients with chronic periodontitis and healthy controls are shown in Table 1. rs2228570 was genotyped by matrix-assisted laser desorption/ionization time-of-flight-mass spectrometry and the genotype and allele frequency distributions were consistent with Hardy-Weinberg equilibrium (Table 2). No significant difference in allelic or genotypic frequencies distributions was found between moderate (Table 3) and severe (Table 4) $\mathrm{CP}$ patients and controls.

Similar negative results were obtained after adjusting for gender and age. The results revealed no statistically significant association between rs2228570 and CP in the population studied.

Table 1. Demographic and clinical characteristics of the studied population.

\begin{tabular}{|c|c|c|c|c|c|c|}
\hline Subject group & $\mathrm{N}$ & Age (years) & Male & Female & $\mathrm{PD}(\mathrm{mm})$ & $\mathrm{CAL}(\mathrm{mm})$ \\
\hline Control & 324 & $50.4 \pm 4.6$ & 195 & 129 & $2.1 \pm 0.2$ & NA \\
\hline Moderate & 352 & $49.9 \pm 6.7$ & 225 & 127 & $4.4 \pm 0.4$ & $4.9 \pm 0.8$ \\
\hline Severe & 88 & $46.6 \pm 4.9$ & 45 & 43 & $6.6 \pm 0.7$ & $7.0 \pm 0.6$ \\
\hline
\end{tabular}

Values are reported as means $\pm \mathrm{SD}$. NA $=$ not applicable.

Table 2. Observed and expected frequencies for genotypes of rs2228570 testing for the goodness of fit to HardyWeinberg equilibrium.

\begin{tabular}{|c|c|c|c|c|c|}
\hline SNP & Genotype/Allele & Observed genotype & Expected genotype & $x^{2}$ & $P$ value \\
\hline \multirow[t]{4}{*}{ rs2228570 $(T \rightarrow C)$} & Genotype $(N=324)$ & & & & \\
\hline & $\mathrm{T} / \mathrm{T}$ & $72(22.2 \%)$ & $71.78(22.2 \%)$ & 0.002 & 0.96 \\
\hline & $\mathrm{C} / \mathrm{T}$ & $161(49.7 \%)$ & $161.44(49.8 \%)$ & & \\
\hline & $\mathrm{C} / \mathrm{C}$ & $91(28.1 \%)$ & $90.78(28.0 \%)$ & & \\
\hline
\end{tabular}

Significant difference was accepted at $\mathrm{P}<0.05$. 
Table 3. Distribution of rs2228570 polymorphism in moderate CP patients and healthy controls.

\begin{tabular}{|c|c|c|c|c|c|c|}
\hline Genotype/allele & $\begin{array}{c}\text { Moderate CP } \\
\text { Patients }[\mathrm{N}=352(\%)]\end{array}$ & Controls [N = $324(\%)]$ & Crude OR $(95 \% \mathrm{Cl})^{\dagger}$ & $P$ value ${ }^{\ddagger}$ & Adjusted OR $(95 \% \mathrm{Cl})^{\mathrm{a}}$ & P value ${ }^{\ddagger}$ \\
\hline$T / T^{*}$ & & $74(21.0)$ & $72(22.2)$ & 1 & & 1 \\
\hline $\mathrm{C} / \mathrm{T}$ & $176(50.0)$ & $161(49.7)$ & $1.064(0.721-1.569)$ & 0.756 & $1.013(0.634-1.617)$ & 0.957 \\
\hline $\mathrm{C} / \mathrm{C}$ & $102(29.0)$ & $91(28.1)$ & $1.091(0.709-1.677)$ & 0.693 & $1.143(0.513-2.574)$ & 0.744 \\
\hline$T^{*}$ & $324(46.0)$ & $305(47.1)$ & 1 & & 1 & \\
\hline C & $380(54.0)$ & $343(52.9)$ & $1.043(0.842-1.292)$ & 0.700 & $1.021(0.568-1.834)$ & 0.773 \\
\hline
\end{tabular}

*Homozygous genotypes were used as reference group. aAdjusted for gender and age. ${ }^{\text {}} \mathrm{OR}$, odds ratio; $\mathrm{Cl}$, confidence interval. ¥Significant difference was accepted at $\mathrm{P}<0.05$.

\begin{tabular}{|c|c|c|c|c|c|c|}
\hline Genotype/allele & $\begin{array}{c}\text { Severe CP } \\
\text { Patients }[\mathrm{N}=88(\%)]\end{array}$ & $\begin{array}{c}\text { Controls } \\
{[\mathrm{N}=324(\%)]}\end{array}$ & Crude OR $(95 \% \mathrm{Cl})^{\dagger}$ & $P$ value ${ }^{\ddagger}$ & Adjusted OR $(95 \% \mathrm{Cl})^{\mathrm{a}}$ & $P$ value ${ }^{\ddagger}$ \\
\hline$T / T^{*}$ & & $21(23.9)$ & $72(22.2)$ & 1 & & \\
\hline $\mathrm{C} / \mathrm{T}$ & $42(47.7)$ & $161(49.7)$ & $0.894(0.494-1.168)$ & 0.712 & $0.850(0.411-1.754)$ & 0.659 \\
\hline $\mathrm{C} / \mathrm{C}$ & $25(28.4)$ & $91(28.1)$ & $0.942(0.488-1.818)$ & 0.858 & $0.915(0.266-3.139)$ & 0.887 \\
\hline$T^{*}$ & $84(47.7)$ & $305(47.1)$ & 1 & & & \\
\hline C & $92(52.3)$ & $343(52.9)$ & $0.974(0.698-1.360)$ & 0.877 & $0.918(0.357-2.359)$ & 0.859 \\
\hline
\end{tabular}

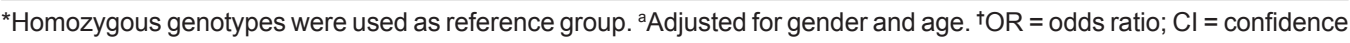
interval. ¥Significant difference was accepted at $\mathrm{P}<0.05$.

\section{DISCUSSION}

$\mathrm{CP}$, which is characterized by inflammatory destruction of connective tissues and alveolar bone, is initiated and perpetuated by a group of predominantly Gram-negative and anaerobic bacteria. It is well-accepted that bacteria are insufficient for CP progression and outcomes, although bacteria are essential for the initiation of CP. The severity of periodontal destruction is significantly modified by genetic and environmental risk factors (Engebretson et al., 1999). SNP rs2228570, located at the translation initiation site of exon 2 in the $5^{\prime}$ coding region in the VDR gene, is a T/C transition generating a shorter peptide product that is 3 amino acids shorter than that of the full-length protein. However, the shorter VDR exhibits higher transcriptional activity (Alimirah et al., 2011) and transmits signals related to bone resorption and inflammation more efficiently compared with the normal protein (Whitfield et al., 2001), which may impact the susceptibility to CP. Therefore, we analyzed the correlation between the alleles and genotypes of rs2228570 and the severity of $\mathrm{CP}$ in a Han Chinese population.

The results of studies examining the impact of rs2228570 are controversial. An Abf haplotype containing rs2228570 has been reported to confer an elevated risk of severe CP in Japanese men (Naito et al., 2007), while several other studies found no significant association between rs2228570 and CP susceptibility (Tachi et al., 2003; Wang et al., 2009; Chen et al., 2012; Tanaka et al., 2013). No significant association was found in a previous meta-analysis of 15 studies including 1338 cases and 1302 controls between rs2228570 and CP (Chen et al., 2012). The present study also revealed no statistically significant association between rs2228570 and CP susceptibility in our population.

Many factors may account for the observed discrepancies among these studies. The racial and environmental differences among the populations may be a highly significant factor. Genetic polymorphisms such as SNPs may interact with environmental factors to influence disease susceptibility. Some environmental factors are essential for disease occurrence. For example, it 
has been reported that low dietary calcium intake can lead to more severe periodontal disease. Moreover, vitamin D and calcium were found to exert effects that were not limited to the spine and hip bones (Hildebolt, 2005). Therefore, not taking into account the vitamin D and calcium intake of all the participants and other environmental factors may have led to false results. Second, rs 2228570 may not influence the phenotype of CP directly, but may be in LD with other (unidentified) diseasecausing polymorphisms elsewhere in VDR or in neighboring genes. However, rs2228570 was not found to be in LD with other SNPs in VDR in populations from Gambia, Britain, Norway, Finland, and Rumania (Nejentsev et al., 2004).

The selection criteria of the studied population may have also influenced the results. Differences between individual studies are also evident in the selection of patients with chronic periodontitis when different criteria were used. Younger controls can lead to selection bias (Page and Beck, 1997). Therefore, we selected controls slightly older than CP patients in order to reduce analysis bias. Smoking has been identified as a major environmental risk factor associated with increased incidence and severity of periodontitis (Grossi et al., 1995); sometimes, the genetic association with periodontitis was evident only when smokers were excluded, confirming the importance of this risk factor (Haber et al., 1993; Schenkein et al., 1995) and suggesting that its effect is strong enough to be seen even in subjects who were not genetically predisposed to periodontitis. Therefore, smokers and smokeless tobacco users were excluded from the present study.

Complex diseases are generally related to variations in multiple genes, each exerting minor effects on the susceptibility or progression of the disease. Additionally, the number and type of functional genes may differ in different ethnic groups (Kinane and Hart, 2003). Numerous studies have demonstrated that no single gene is sufficient for the occurrence of CP. Therefore, CP is affected by more than one gene, and identifying genes related to CP may facilitate risk assessment and prevention. Further studies should extensively analyze gene-gene and gene-environment interactions using different ethnic groups and a larger number of samples to obtain a better insight into the pathogenesis of CP. Two or more SNPs should be investigated simultaneously, perhaps by haplotype analysis (Nikolopoulos et al., 2008).

In conclusion, the results of this study revealed no statistically significant association between rs2228570 and CP in our population.

\section{Conflicts of interest}

The authors declare no conflict of interest.

\section{ACKNOWLEDGMENTS}

Research supported by the Natural Science Foundation of Shanghai (Grant\#10ZR1425900) and the Shanghai Health Bureau (Grant \#2009202), Shanghai, China.

\section{REFERENCES}

Alimirah F, Peng X, Murillo G and Mehta RG (2011). Functional significance of vitamin D receptor Fokl polymorphism in human breast cancer cells. PloS One 6: e16024.

Armitage GC (1999). Development of a classification system for periodontal diseases and conditions. Ann. Periodontol. 4: 1-6. Chen LL, Li H, Zhang PP and Wang SM (2012). Association between vitamin D receptor polymorphisms and periodontitis: a meta-analysis. J. Periodontol. 83: 1095-1103.

Descroix V, Kato S, Lézot F and Berdal A (2010). Physiopathology of dental rickets in vitamin D receptor-ablated mice. J. Dent. 
Res. 89: 1427-1432.

Eke PI, Page RC, Wei L, Thornton-Evans G, et al. (2012). Update of the case definitions for population-based surveillance of periodontitis. J. Periodontol. 83: 1449-1454.

Engebretson SP, Lamster IB, Herrera-Abreu M, Celenti RS, et al. (1999). The influence of interleukin gene polymorphism on expression of interleukin-1 beta and tumor necrosis factor-alpha in periodontal tissue and gingival crevicular fluid. $J$. Periodontol. 70: 567-573.

Grossi SG, Genco RJ, Machtei EE, Ho AW, et al. (1995). Assessment of risk for periodontal disease. II. Risk indicators for alveolar bone loss. J. Periodontol. 66: 23-29.

Haber J, Wattles J, Crowley M, Mandell R, et al. (1993). Evidence for cigarette smoking as a major risk factor for periodontitis. J. Periodontol. 64: 16-23.

Hildebolt CF (2005). Effect of vitamin D and calcium on periodontitis. J. Periodontol. 76: 1576-1587.

Kinane DF and Hart TC (2003). Genes and gene polymorphisms associated with periodontal disease. Crit. Rev. Oral Biol. Med. 14: 430-449.

Kinane DF, Shiba H and Hart TC (2005). The genetic basis of periodontitis. Periodontol. 2000 39: 91-117.

Michalowicz BS, Diehl SR, Gunsolley JC, Sparks BS, et al. (2000). Evidence of a substantial genetic basis for risk of adult periodontitis. J. Periodontol. 71: 1699-1707.

Nagpal S, Na S and Rathnachalam R (2005). Noncalcemic actions of vitamin D receptor ligands. Endocr. Rev. 26: 662-687.

Naito M, Miyaki K, Naito T, Zhang L, et al. (2007). Association between vitamin D receptor gene haplotypes and chronic periodontitis among Japanese men. Int. J. Med. Sci. 4: 216-222.

Nares S (2003). The genetic relationship to periodontal disease. Periodontol. 2000 32: 36-49.

Nejentsev S, Godfrey L, Snook H, Rance H, et al. (2004). Comparative high-resolution analysis of linkage disequilibrium and tag single nucleotide polymorphisms between populations in the vitamin D receptor gene. Hum. Mol. Genet. 13: 1633-1639.

Nikolopoulos GK, Dimou NL, Hamodrakas SJ and Bagos PG (2008). Cytokine gene polymorphisms in periodontal disease: a meta-analysis of 53 studies including 4178 cases and 4590 controls. J. Clin. Periodontol. 35: 754-767.

OMIM (2004). Online Mendelian Inheritance in Man. Available at [http://omim.org/entry/601769\#35]. Accessed December 14, 2014.

Page RC and Beck JD (1997). Risk assessment for periodontal diseases. Int. Dent. J. 47: 61-87.

Schaeffeler E, Zanger UM, Eichelbaum M, Asante-Poku S, et al. (2008). Highly multiplexed genotyping of thiopurine s-methyltransferase variants using MALD-TOF mass spectrometry: reliable genotyping in different ethnic groups. Clin. Chem. 54: 1637-1647.

Schenkein HA, Gunsolley JC, Koertge TE, Schenkein JG, et al. (1995). Smoking and its effects on early-onset periodontitis. J. Am. Dent. Assoc. 126: 1107-1113.

Stabholz A, Soskolne WA and Shapira L (2010). Genetic and environmental risk factors for chronic periodontitis and aggressive periodontitis. Periodontol. 2000 53: 138-153.

Tachi Y, Shimpuku H, Nosaka Y, Kawamura T, et al. (2003). Vitamin D receptor gene polymorphism is associated with chronic periodontitis. Life Sci. 73: 3313-3321.

Tanaka K, Miyake Y, Hanioka T and Arakawa M (2013). VDR gene polymorphisms, interaction with smoking and risk of periodontal disease in Japanese women: the Kyushu Okinawa maternal and child health study. Scand. J. Immunol. 78: 371-377.

Uitterlinden AG, Fang Y, Van Meurs JB, Pols HA, et al. (2004). Genetics and biology of vitamin D receptor polymorphisms. Gene 338: 143-156.

Wang C, Zhao H, Xiao L, Xie C, et al. (2009). Association between vitamin D receptor gene polymorphisms and severe chronic periodontitis in a Chinese population. J. Periodontol. 80: 603-608.

Whitfield GK, Remus LS, Jurutka PW, Zitzer H, et al. (2001). Functionally relevant polymorphisms in the human nuclear vitamin D receptor gene. Mol. Cell Endocrinol. 177: 145-159. 